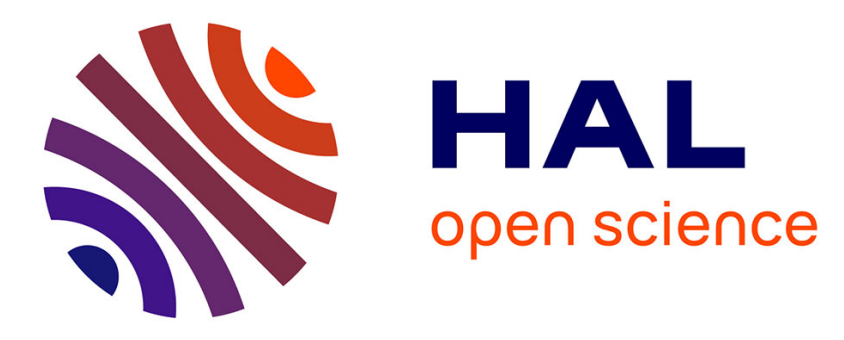

\title{
Thermomechanical analysis of the crack tip zone in stretched crystallizable natural rubber by using infrared thermography and digital image correlation
}

\author{
Jose Ricardo Samaca Martinez, Xavier Balandraud, Evelyne Toussaint, \\ Jean-Benoit Le Cam, Daniel Berghezan
}

\section{To cite this version:}

Jose Ricardo Samaca Martinez, Xavier Balandraud, Evelyne Toussaint, Jean-Benoit Le Cam, Daniel Berghezan. Thermomechanical analysis of the crack tip zone in stretched crystallizable natural rubber by using infrared thermography and digital image correlation. Polymer, 2014, 55 (24), pp.6345-6353. 10.1016/j.polymer.2014.10.010 . hal-01148251

\section{HAL Id: hal-01148251 \\ https://hal.science/hal-01148251}

Submitted on 28 Jan 2019

HAL is a multi-disciplinary open access archive for the deposit and dissemination of scientific research documents, whether they are published or not. The documents may come from teaching and research institutions in France or abroad, or from public or private research centers.
L'archive ouverte pluridisciplinaire HAL, est destinée au dépôt et à la diffusion de documents scientifiques de niveau recherche, publiés ou non, émanant des établissements d'enseignement et de recherche français ou étrangers, des laboratoires publics ou privés. 


\title{
Thermomechanical analysis of the crack tip zone in stretched crystallizable natural rubber by using infrared thermography and digital image correlation
}

\author{
J.R. Samaca Martinez ${ }^{\text {a, b, c }}$, X. Balandraud ${ }^{\text {b, d, }{ }^{*}, \text { E. Toussaint }}{ }^{\text {a, b }}$, J.-B. Le Cam ${ }^{\text {e }}$, D. Berghezan ${ }^{c}$
}

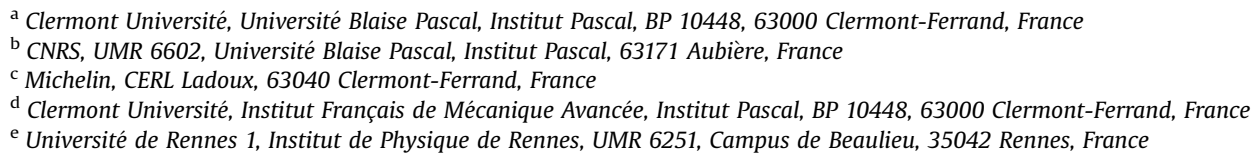

This paper provides the first characterization of heat source field in the crack tip zone in carbon black filled natural rubber (NR). It focuses more especially on the calorific effects of strain induced crystallization (SIC). For this purpose, full thermal and kinematic fields are measured simultaneously. Initial image processing based on motion compensation enables us to track the temperature of any material point at the specimen surface. A second image processing stage, based on the heat diffusion equation, enables us to obtain the fields of heat sources produced and absorbed by the material during the test. The heterogeneity of the stretch states is analyzed from the kinematic measurements. In terms of heat production, crystallization acts in two opposite ways in the crack tip zone: the crystallization process produces additional heat, but crystallites act as fillers, which increases material stiffness in the crack tip zone. Moreover, the heat sources in the crack tip zone remain positive and small during unloading. This phenomenon is due to the production of mechanical dissipation and probably a continuation of the crystallization process. The results attained are compared with those recently obtained in noncrystallizing carbon black filled styrene butadiene rubber (SBR50).

\section{Introduction}

Fracture mechanics in rubber is classically addressed through phenomenological approaches, especially through the strain energy release rate, also named tearing energy [1]. The tearing energy is the total energy necessary to create a unit fracture surface by crack propagation. To account for the physical phenomena involved in crack growth, the expression of the tearing energy can be modified, for instance by weighting it with a dissipative energy function to model the effects of dissipation at the crack tip [2]. Despite this, such approaches remain a description of fracture on the global scale and do not enable us to satisfactorily predict crack

* Corresponding author. Clermont Université, Institut Français de Mécanique Avancée, Institut Pascal, BP 10448, 63000 Clermont-Ferrand, France. Fax: +33 473 288100.

E-mail address: xavier.balandraud@ifma.fr (X. Balandraud). propagation paths or to understand how changes in the microstructure affect crack growth. Consequently, the investigation of physical mechanisms that drive crack growth is an essential prerequisite to improving fracture mechanics theories.

This explains why, over the last two decades, the state of the matter in the crack tip zone has increasingly been investigated. For instance, Refs. [3-6] have used wide-angle x-ray diffraction (WAXD) to map the crystallinity in the crack tip zone in natural rubber. Cavitation has also been identified as a phenomenon of paramount importance in the crack growth mechanism [7,8]. More recently, the interaction between cavitation and SIC has been highlighted and investigated in Ref. [9]. Finally, several authors have proposed mechanisms of crack growth [7,10,11]. Even though the results obtained have increased our knowledge of crack growth mechanisms, it is currently not possible to update constitutive models in order to account for all these phenomena and their interactions. Therefore, between a global description of fracture with continuum quantities (for instance tearing energy) and the 
description of the physical mechanisms at the local scale, we think that a trade-off can be found by measuring continuum quantities at the scale at which phenomena take place, i.e. in the crack tip zone. This should be all the more interesting since the continuum quantities measured are energies (making the link with tearing energy).

In our previous work, we investigated the thermomechanical response and calorific effects during the homogeneous deformation of rubber, using full-field measurement techniques. We highlighted the calorimetric signature of various phenomena involved during deformation: entropic and isentropic elasticity [12], viscosity due to filler adding [13], SIC [14,15] and the Mullins effect [16]. Then we studied heterogeneous deformations by developing a new data processing methodology in the case of a large displacement of points at the observed surface [17]. This methodology was applied to the determination of the kinematic and thermal fields in the crack tip zone of filled styrene butadiene rubber (SBR) to evaluate the effects of the mechanical dissipation of the total heat source $^{1}$ : see Ref. [18]. In the present study, the effects of SIC on the full kinematic, thermal and heat source fields are investigated in the crack tip zone by using such methodology. The objective is therefore to link the change in the microstructure with variation in the heat sources, which are quantities originating in continuum mechanics.

Section 2 of the paper describes the experimental setup. Section 3 presents the image processing used to assess heat sources from temperature fields measured at the specimen surface in the case of large heterogeneous deformation. Section 4 gives the results and the analysis of the full kinematic, thermal and heat source fields and compares the results with those obtained in SBR material, which is not crystallizable under strain.

\section{Experimental setup}

\subsection{Material and specimen geometry}

The material used in the present study was a crystallizable natural rubber filled with 50 parts per hundred of rubber in weight (phr) of carbon black. It is denoted NR50 in the following. Table 1 summarizes its chemical composition. It was cured for $22 \mathrm{~min}$ at $150{ }^{\circ} \mathrm{C}$.

The geometry of the specimens is presented in Fig. 1. The width, height and thickness were equal to $80 \mathrm{~mm}, 13 \mathrm{~mm}$ and $2 \mathrm{~mm}$, respectively. This corresponds to classic Pure Shear (PS) geometry. The specimens were notched at one of their sides using a razor blade prior to testing. The initial crack length was about $8 \mathrm{~mm}$. This undeformed state geometry was considered as the reference configuration in the present study.

\subsection{Loading conditions}

The mechanical test corresponded to uniaxial tensile loading. One load-unload cycle was applied under imposed displacement using a $500 \mathrm{~N}$ INSTRON 5543 testing machine (see Fig. 2). The signal shape was triangular in order to ensure a global constant strain rate during loading and unloading. The maximum global stretch ratio $\lambda_{g}$ was equal to 1.5. The global loading rate and the global strain rate were equal to $\pm 200 \mathrm{~mm} / \mathrm{min}$ and $\pm 0.26 \mathrm{~s}^{-1}$, respectively.

\footnotetext{
1 'Heat source' is used in this paper to designate the heat power density (in W/ $\mathrm{m}^{3}$ ) that is produced or absorbed by the material. Note that 'heat source' and 'heat' must be distinguished: heat (in $\mathrm{J} / \mathrm{m}^{3}$ ) is the temporal integration of heat source.
}

Table 1

Chemical composition.

\begin{tabular}{lc}
\hline Ingredient & Amount (phr) \\
\hline Natural rubber NR & 100 \\
Carbon black & 50 \\
Antioxidant 6PPD & 1.9 \\
Stearic acid & 2 \\
Zinc oxide ZnO & 2.5 \\
Accelerator CBS & 1.6 \\
Sulfur solution 2H & 1.6 \\
\hline
\end{tabular}

$\rightarrow\{2 \mathrm{~mm}$

(a)

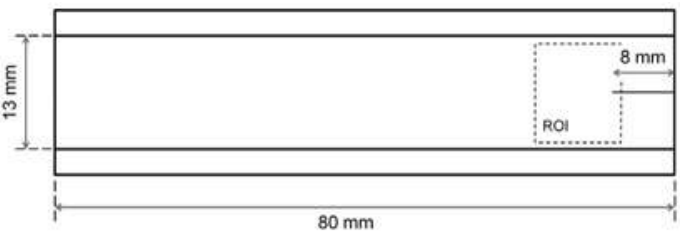

(b)
Fig. 1. Specimen geometry. a) Side view. b) Front view.

\subsection{Displacement field measurements}

The kinematic fields were measured using the Digital Image Correlation (DIC) technique, which consists of correlating the grey levels between two different images of an observed region of the specimen surface named "Region Of Interest" (ROI) (see for instance Refs. [19-21]). Images were recorded using a cooled 16-bit PCO-Edge sCMOS camera. The sensor of the camera features $2650 \times 2160$ pixels. The acquisition frequency $f_{a}$ was set to $50 \mathrm{~Hz}$. Uniform lighting of the specimen surface was ensured by a set of three fixed cold lamps in order not to heat the specimen and not to induce external radiations. In order to improve the contrast of the images, white talc was deposited on the surface of the specimen before the measurements. Particular attention was paid to these preliminary settings for a maximum use of the dynamics of the camera. The software employed for the correlation process was SeptD [22]. A set of sub-images was considered to determine the displacement field of a given image with respect to a reference image. Each sub-image is referred to as a "Zone of Interest" (ZOI). A bilinear correlation function was employed to calculate the displacement of the center of a given ZOI between the two images. Correlation parameters were chosen to reach a spatial resolution of 10 pixels, corresponding to $101 \mu \mathrm{m}$.

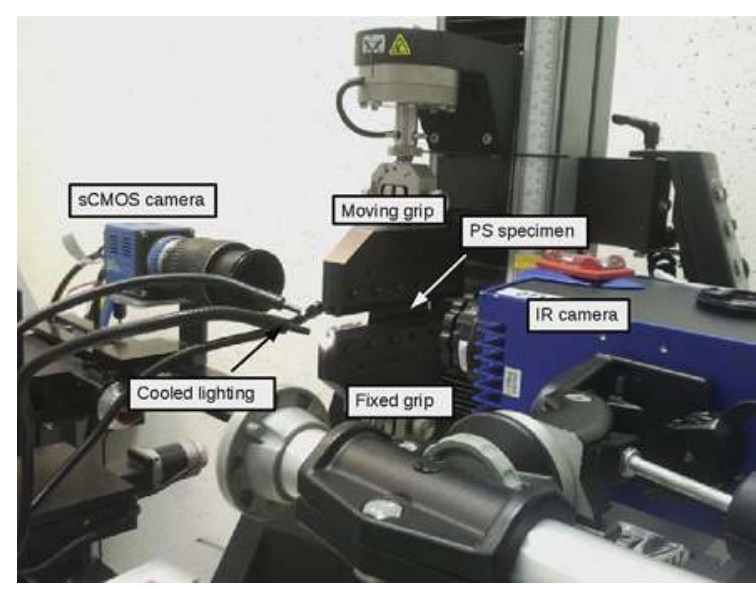

Fig. 2. Experimental device. 


\subsection{Temperature field measurements}

Temperature measurements were carried out using a Cedip Jade III-MWIR infrared (IR) camera, featuring a focal plane array of $320 \times 240$ pixels and detectors with a wavelength range of $3.5-5 \mu \mathrm{m}$. The Noise Equivalent Temperature Difference reported on the data sheet of the camera is $0.02{ }^{\circ} \mathrm{C}$. The integration time was set to $1500 \mu \mathrm{s}$. The same acquisition frequency $f_{a}$ as for the kinematic measurements was chosen: $50 \mathrm{~Hz}$. In fact, each recorded image is the average of two successive images captured at $100 \mathrm{~Hz}$. This procedure enables us to improve the resolution of the measurement. The calibration of the camera detectors was performed using a black body and employing a two-point Non-Uniformity Correction (NUC) procedure. The spatial resolution of the temperature fields is the size of the pixel on the surface of the specimen. It was here equal to $62.5 \mu \mathrm{m}$.

The thermal quantity used in the present study is actually the temperature variation with respect to the initial temperature. The field of the temperature variation was obtained by using a specific image processing technique presented in the following section.

\section{Image processing}

This section details several aspects of the image processing technique performed on the thermal and kinematic measurements: motion compensation procedure (Section 3.1), heat source reconstruction (Section 3.2), and processing of the kinematic measurements (Section 3.3).

\subsection{Motion compensation technique}

The aim of the motion compensation technique is to be able to follow the temperature variation at any material point on the specimen surface. The output will be used further to construct the heat source field in the reference configuration, which was chosen here as the undeformed state, by using the heat diffusion equation (see Section 3.2). Since the specimen undergoes a large deformation and the thermal fields are captured in the (deformed) current configuration, the correspondence between the current geometry and the reference geometry must be implemented. Thanks to the movement compensation, any current thermal field can be mapped in the reference configuration. The technique was initially developed in Ref. [12] using only thermal fields, then was improved in Ref. [17], using both kinematic and thermal fields. The methodology employed for this latter method is briefly recalled below.

The principle is based on the simultaneous measurement of the kinematic and thermal fields at the specimen surface using two cameras on opposite sides of the specimen (see Fig. 2). As the two cameras do not observe the same geometric zone, it is therefore necessary to identify a common point in both images. Then, displacement fields are calculated on the thermal grid by the interpolation of the kinematic grid on the thermal one. These new displacement fields enable us to place the temperature values back in the reference configuration. Finally, the calculation of heat sources relies on the temperature variations $\theta$.

In case of large displacements of material points at the surface observed by the IR camera, a specific procedure must be employed to subtract the reference temperature $T_{0}$. IR cameras are characterized by the non-uniformity of their detector matrices [23,24]. The impact of this non-uniformity can be limited by performing a NonUniformity Correction (NUC) using a black body. After NUC, the measurements of homogeneous temperature fields remain altered by a small spatial heterogeneity [12]. As an example, Fig. 3-a shows the homogeneous temperature field measured by the IR camera: a small heterogeneity is observed. If the camera is run for several hours before performing the calibration and the measurement, this (a)

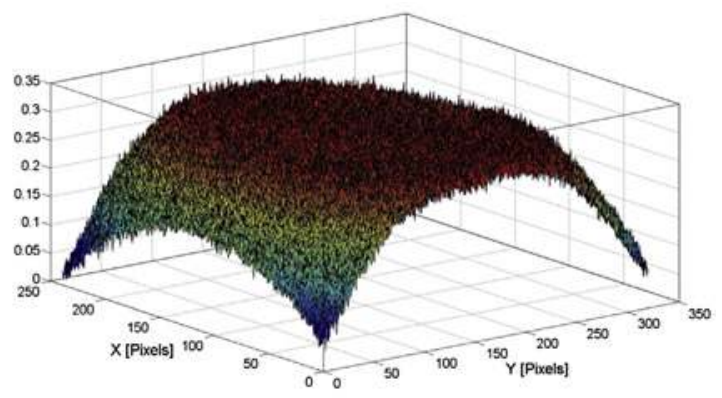

(b)

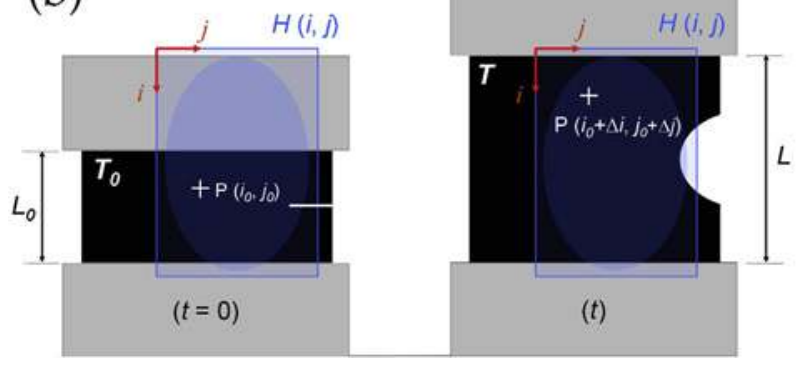

Fig. 3. Motion compensation technique. a) Temperature field $H$. b) Situation in the case of large displacements of the material points.

heterogeneity remains constant over time $t$. Here, the amplitude of the heterogeneity is about three tenths of a degree. Let us denoted it by $H(i, j, t)=H(i, j)$, where $i$ and $j$ are the pixel coordinates in the image. This property is very useful for correctly measuring temperature variations when there is no displacement of the observed surface. Indeed, in this case the temperature variation at a given point $P$ is simply obtained by subtracting the initial temperature from the current one measured by the camera at this point. However, heterogeneity $H$ may have an important consequence in the case of large displacements, as it is the case in the present study when performing tests on rubber materials. Let us examine the situation for a material point $P$, which is located at pixel $\left(i_{0} j_{0}\right)$ on the specimen surface in the reference configuration, as shown in Fig. 3-b. The temperature $T_{0 \text { meas }}\left(i_{0}, i_{0}\right)$ which is measured at this point at time $t=0$ is equal to the sum of the actual reference temperature $T_{0}\left(i_{0}, i_{0}\right)$ and the additional value $H\left(i_{0}, j_{0}\right)$ :

$T_{0 \text { meas }}\left(i_{0}, j_{0}\right)=T_{0}\left(i_{0}, j_{0}\right)+H\left(i_{0}, j_{0}\right)$

Thus at time $t$, point $P$ is located at a new pixel $\left(i_{0}+\Delta i j_{0}+\Delta j\right)$ of the grid of the IR camera, where $\Delta i$ and $\Delta j$ are the components of the displacement vector of point $P$ measured in pixels. The temperature measured by the camera at this new pixel is equal to:

$T_{\text {meas }}\left(i_{0}+\Delta i, j_{0}+\Delta j\right)=T\left(i_{0}+\Delta i, j_{0}+\Delta j\right)+H\left(i_{0}+\Delta i, j_{0}+\Delta j\right)$

The aim now is to define a procedure to obtain the temperature variation $\theta_{P}$ at this point $P$ :

$\theta_{P}=T\left(i_{0}+\Delta i, j_{0}+\Delta j\right)-T_{0}\left(i_{0}, j_{0}\right)$

Let us subtract equation (1) from equation (2). This leads to:

$$
\begin{aligned}
& T_{\text {meas }}\left(i_{0}+\Delta i, j_{0}+\Delta j\right)-T_{0 \text { meas }}\left(i_{0}, j_{0}\right) \\
& =\theta_{P}+H\left(i_{0}+\Delta i, j_{0}+\Delta j\right)-H\left(i_{0}, j_{0}\right)
\end{aligned}
$$

This equation shows that the temperature variation $\theta_{P}$ at point $P$ cannot be obtained by subtracting the two values measured by the camera: 


$$
T_{\text {meas }}\left(i_{0}+\Delta i, j_{0}+\Delta j\right)-T_{0 \text { meas }}\left(i_{0}, j_{0}\right) \neq \theta_{P}
$$

The amplitude of the heterogenity $H$ is a few tenths of a degree. This can be considered as a problem if the temperature variation $\theta_{P}$ has the same order of magnitude or less. In such a case, the solution consists of subtracting field $H$ from the reference temperature field and from all the temperature fields, all measured by the IR camera. This procedure must be carried out before performing the motion compensation technique.

\subsection{Heat source reconstruction from IR measurements}

The temperature variations are the consequence of the heat produced or absorbed by the material during loading. The reconstruction of the heat source fields from the temperature variation fields is based on the heat diffusion equation. Two points are discussed below: first, a two-dimensional (2D) version of this equation that can be defined for the specimen used in the present study; second, some filtering operations that must be applied as the thermal input data are unavoidably noisy.

\subsubsection{Two-dimensional version of the heat diffusion equation}

This section recalls how heat sources can be calculated in the reference configuration from $2 \mathrm{D}$ temperature measurements obtained at the surface of a thin specimen. For this purpose, the threedimensional formulation of the heat diffusion equation is simplified to a $2 \mathrm{D}$ formulation by taking into account some usual assumptions. These assumptions are briefly recalled here.

For further information the reader can refer to Ref. [25]. The heat diffusion equation can be written as follows in the Lagrangian configuration:

$$
\rho_{0} C_{E, V_{k}} \frac{\mathrm{d} T}{\mathrm{~d} t}-\operatorname{Div}\left(K_{0} \operatorname{Grad} T\right)-R=\underbrace{D_{1}+\rho_{0} T \frac{\partial^{2} \Psi}{\partial T \partial E} \dot{E}+\rho_{0} T \frac{\partial^{2} \Psi}{\partial T \partial V_{k}} \dot{V}_{k}}_{S}
$$

where:

- $\Psi$ is the specific free energy potential, which is a function of the temperature $T$, a measure $E$ of the deformation, and other state variables $V_{k}$ characterizing the state of the material. For instance the fraction of crystallites can be introduced as a state variable in order to model the part of the heat source caused by a change in the degree of crystallization;

- $R$ is the external heat source by radiation;

- $S$ is the total heat source produced by the material itself due to deformation;

- $\rho_{0}$ is the density, $K_{0}$ is the thermal conductivity tensor and $C_{E, V_{k}}$ is the specific heat at constant $E$ and $V_{k}$.

The right member contains the different heat sources produced or absorbed by the material. It is composed of two parts: thermomechanical couplings and mechanical dissipation $D_{1}$ (also named intrinsic dissipation). It can be noted that this thermomechanical framework does not require to postulate the state variables, the specific free energy function and the mechanical dissipation to be able to calculate the evolution of the total heat source from the temperature changes.

Some hypotheses will now be introduced:

\section{- Assumption No. 1}

The heat conduction tensor is considered as isotropic: $K_{0 i j}=k_{0} \delta_{i j}$, where $k_{0}$ is the thermal conductivity coefficient.
Consequently, the heat diffusion equation (6) can be rewritten as follows:

$\rho_{0} C_{E, V_{k}} \frac{\mathrm{d} T}{\mathrm{~d} t}-k_{0} \Delta T-R=S$

where $\Delta$ is the Laplacian operator in the Lagrangian configuration.

\section{- Assumption No. 2}

The temperature through the specimen thickness is considered as nearly homogeneous. Indeed using thin specimens, the surface temperature is very close to the mean temperature through the thickness. Consequently, a 2D heat diffusion equation can be obtained by integrating equation (7) over the specimen thickness, leading to:

$\rho_{0} C_{E, V_{k}} \frac{\mathrm{d} T}{\mathrm{~d} t}+\frac{T-T_{\mathrm{amb}}}{\tau_{2 \mathrm{D}}}-k_{0} \Delta_{2 \mathrm{D}} T-R=S$

where $\Delta_{2 \mathrm{D}}$ is the Laplacian operator in the specimen plane in the Lagrangian coordinate system. $\tau_{2 \mathrm{D}}$ is a time constant which characterizes the heat exchanges along the $Z$-direction by convection with the air at the specimen surface. It is equal to $40 \mathrm{~s}$ for the present experiments. It can be obtained by identification from an elementary test of natural return to the ambient temperature. $T_{\mathrm{amb}}$ is the ambient temperature, which is nearly equal to the reference temperature field $T_{\text {ref }}$ of the specimen in the reference configuration (undeformed state).

\section{- Assumption No. 3}

The external heat sources $R$ are assumed to be constant over time. As a consequence, equation (8) can be rewritten as:

$\rho_{0} C_{E, V_{k}}\left(\frac{\mathrm{d} \theta}{\mathrm{d} t}+\frac{\theta}{\tau_{2 \mathrm{D}}}\right)-k_{0} \Delta_{2 \mathrm{D}} \theta=S$

where $\theta$ is the 2D temperature variation field: $\theta(X, Y, t)=T(X, Y, t)-T_{\text {ref }}(X, Y)$.

The heat source $S$ is expressed in $W / \mathrm{m}^{3}$. However this quantity can be expressed in ${ }^{\circ} \mathrm{C} / \mathrm{s}$ by dividing it by $\rho_{0} C_{E, V_{k}}$. This unit enables us to directly read the temperature rate that would be observed in the case of adiabatic variation. The temperature rate is obviously different for a non-adiabatic variation. Dividing equation (9) by $\rho_{0} C_{E, V_{k}}$ leads to:

$\frac{\mathrm{d} \theta}{\mathrm{d} t}+\frac{\theta}{\tau_{2 \mathrm{D}}}-D_{0} \Delta_{2 \mathrm{D}} \theta=\frac{S}{\rho_{0} C_{E, V_{k}}}$

where $D_{0}$ is the thermal diffusivity coefficient, which is equal to $k_{0} / \rho_{0} C_{E, V_{k}}$.

The heat source on the right-hand side of equation (10) is calculated by processing the temperature variations appearing on the left-hand side of the equation. Finite differences are applied for the derivative terms: centered two-point derivation for the term $\mathrm{d} \theta / \mathrm{d} t$, and three-point difference along the $X$ - and $Y$-directions to estimate the Laplacian term. Before performing this calculation, some filtering operations are necessary.

\subsubsection{Filtering}

As the measured temperature variations are noisy, filtering must be carried out. Temperature variation maps are spatially and temporally filtered using averaging filters to obtain a better resolution of the measurement. However, these filtering processes 
must be adjusted in order not to impair the temporal and spatial resolutions. Table 2 gives the size of the mean filters as well as the new temporal and spatial resolutions for each term of the lefthand side of equation (10). It can be deduced that the spatial and the temporal resolution of the heat source fields are equal to $0.44 \mathrm{~mm}(5+2=7$ pixels $)$ and $0.14 \mathrm{~s}(5+2=7$ time steps $)$, respectively.

\subsection{Processing of the kinematic measurements}

From the horizontal and vertical displacement fields obtained by the kinematic measurements, some quantities are deduced: the first and second invariants $I_{1}$ and $I_{2}$ of the left Cauchy-Green tensor $\mathbf{B}$, the maximum stretch ratio $\lambda_{\max }$ and the biaxiality ratio $b$. These quantities are used in the present study to characterize the heterogeneity of the stretch states in the ROI observed by the camera. Their definitions are recalled below.

The Cauchy-Green tensor is defined by $\mathbf{B}=\mathbf{F} \mathbf{F}^{t}$, where $\mathbf{F}$ is the deformation gradient tensor. The three principal stretch ratios $\left(\lambda_{1}, \lambda_{2}, \lambda_{3}\right)$ are defined as the square roots of the eigenvalues of $\mathbf{B}$. Assuming the material is incompressible, we have $J=\operatorname{det} \mathbf{F}=\lambda_{1} \lambda_{2} \lambda_{3}=1$. The two invariants $I_{1}$ and $I_{2}$ of $\mathbf{B}$ are written as a function of the principal stretch ratios $\lambda_{i}$ as follows:

$\left\{\begin{array}{l}I_{1}=\lambda_{1}^{2}+\lambda_{2}^{2}+\lambda_{3}^{2} \\ I_{2}=\lambda_{1}^{2} \lambda_{2}^{2}+\lambda_{2}^{2} \lambda_{3}^{2}+\lambda_{3}^{2} \lambda_{1}^{2}\end{array}\right.$

For the kinematic analysis of the tests, different types of representations are used to characterize the heterogeneity of the stretch states:

- plotting in the $\left(I_{1}, I_{2}\right)$ plane - Each ZOI in the ROI can be represented by a point in this plane. Note that in this representation, the equibiaxial (ET), pure shear (PS) and uniaxial tensile (UT) states correspond to three curves meeting at point $(3,3)$;

- mapping the stretch states over the ROI - A color code is employed. The ET, PS and UT states are represented by the blue, green and red colors, respectively. Intermediate states between two pure states are represented by intermediate colors using a weighting coefficient between two colors defined by using the distances in the $\left(I_{1}, I_{2}\right)$ plane from the two corresponding curves;

- mapping the maximum stretch ratios over the ROI - The maximum stretch ratio $\lambda_{\max }$ is defined by $\lambda_{\max }=\max \left(\lambda_{1}, \lambda_{2}\right)$;

- mapping the biaxiality ratios over the ROI - The biaxiality ratio $b$ is defined as the ratio between the logarithm of $\lambda_{2}$ and the logarithm of $\lambda_{1}$, where $\lambda_{1}$ and $\lambda_{2}$ are respectively the highest and second highest principal stretch ratios. It is equal to 0 for PS, 1 for ET and -0.5 for UT.

\section{Results and discussion}

Sections 4.1 and 4.2 give the kinematic and calorimetric results for the NR50 specimen. Finally, Section 4.3 presents a comparison with results previously obtained in SBR50 [18].

\section{Table 2}

Size of the averaging filters used for smoothing the temperature variation fields. The consequence on each term of the heat diffusion equation is indicated in terms of temporal and spatial resolutions.

\begin{tabular}{lllll}
\hline Term & $\begin{array}{l}\text { Spatial } \\
\text { filtering }\end{array}$ & $\begin{array}{l}\text { Temporal } \\
\text { filtering }\end{array}$ & $\begin{array}{l}\text { Spatial } \\
\text { resolution }\end{array}$ & $\begin{array}{l}\text { Temporal } \\
\text { resolution }\end{array}$ \\
\hline$\theta / \tau_{2 \mathrm{D}}$ & $3 \times 3$ & No filtering & $0.19 \mathrm{~mm}$ & $0.02 \mathrm{~s}$ \\
$\mathrm{~d} \theta / \mathrm{d} t$ & $3 \times 3$ & 5 & $0.19 \mathrm{~mm}$ & $0.14 \mathrm{~s}$ \\
$-D_{0} \Delta_{2 \mathrm{D}} \theta$ & $5 \times 5$ & No filtering & $0.44 \mathrm{~mm}$ & $0.02 \mathrm{~s}$ \\
\hline
\end{tabular}

\subsection{Kinematic analysis}

Fig. 4 presents the distribution in plane $\left(I_{1}, I_{2}\right)$ of the stretch states at the end of the loading phase. Each point on the graph corresponds to one ZOI. Some comments can be made:

- all the points are located between the PS curve (in green) and the UT curve (in red) (see Fig. 4-a). Only a few points correspond to very high values of $I_{1}$ and $I_{2}$, i.e. to high values of $\lambda_{i}$ in the PS state;

- Fig. 4-b shows that, as expected, the zone which is far from the crack tip is in PS. The zones above and below the crack lips are in UT. The length of the zone of influence of the crack corresponding to the zone that is not in PS in plane $(X, Y)$ is about 7-8 mm;

- very near the crack tip, a PS state is also observed (see Fig. 4-b and $-c$ ). This can be explained by a kinematic state which is actually close to PS in the $(X, Z)$ plane. However, it must be noted that the spatial resolution of the stretch ratio field is $303 \mu \mathrm{m}$ due to centered derivation (three times the spatial resolution of the displacements, see Section 2.3). As a consequence, the measured values of $\left(I_{1}, I_{2}\right)$ are averaged in the zones of high displacement gradient, typically in the crack tip zone, leading to a strong approximation of stretch state.

Fig. 5-a presents the map of the maximum stretch ratio $\lambda_{\max }$ at the end of the loading phase. The maximum stretch ratio is quite homogeneous over the ROI except in the vicinity of the crack tip. Fig. 5-b shows the variation over time of $\lambda_{\max }$ at two different points: point A located at the crack tip, and point $B$ located far from the zone of influence of the crack. Two comments can be made from this figure:

- at point A (corresponding to a ZOI of $101 \times 101 \mu \mathrm{m}^{2}$ ), $\lambda_{\max }$ is equal to 5.1 at the end of loading phase. The curve shape is not symmetrical between loading and unloading. In particular, $\lambda_{\max }$ decreases slowly from 5.1 to 4.5 . This dissymmetry can be explained either by the Mullins effect which occurs during the loading phase, or by a dissymmetry in the kinetics of crystallization and crystallite melting.

- as expected, the variation over time of $\lambda_{\max }$ at point $\mathrm{B}$ follows the imposed loading.

Calorimetric analysis is expected to provide complementary information on these phenomena.

\subsection{Calorimetric analysis}

Let us first analyze the temperature variation $\theta$ during the test:

- Fig. 6-a presents the map of $\theta$ at the end of the loading phase. It can be observed that the distribution is not exactly symmetrical with respect to the crack direction. The maximum value of $\theta$ $\left(1.8{ }^{\circ} \mathrm{C}\right)$ is located slightly above the crack tip. The highest temperature variations cover a zone around the tip, as well as above and below;

- Fig. 6-b shows the variation in $\theta$ for point A, which corresponds to the crack tip. The temperature increases monotonously during the loading phase, and decreases nearly symmetrically during unloading. A return to $\theta=0$ is observed at the end of unloading.

As the temperature fields depend on heat diffusion, it is necessary to examine the heat sources for further analysis. Fig. 7-a shows the heat source map at the end of the loading phase. The 
(a)

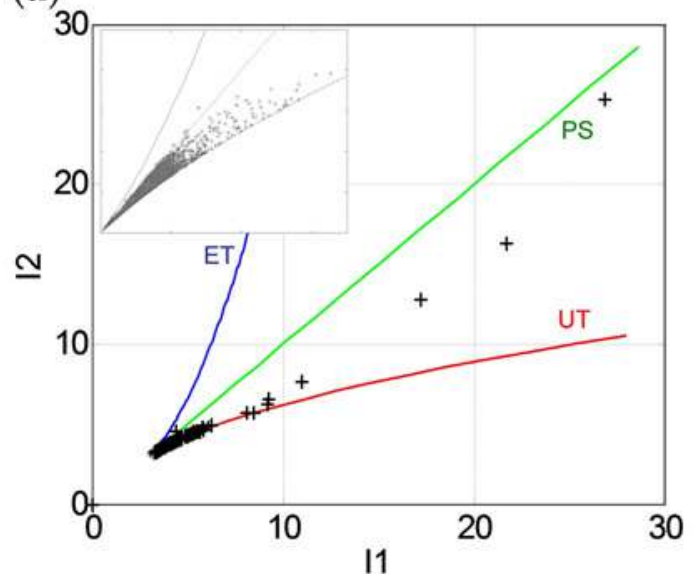

(b) 2

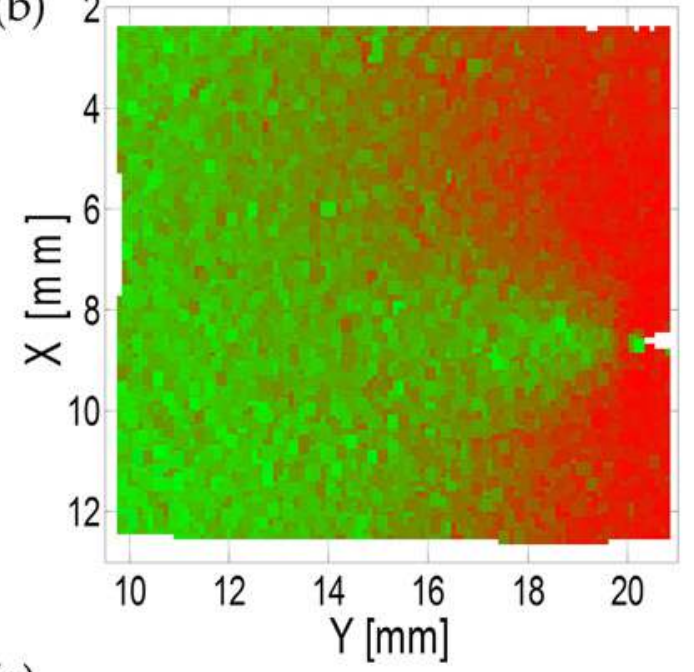

(c)

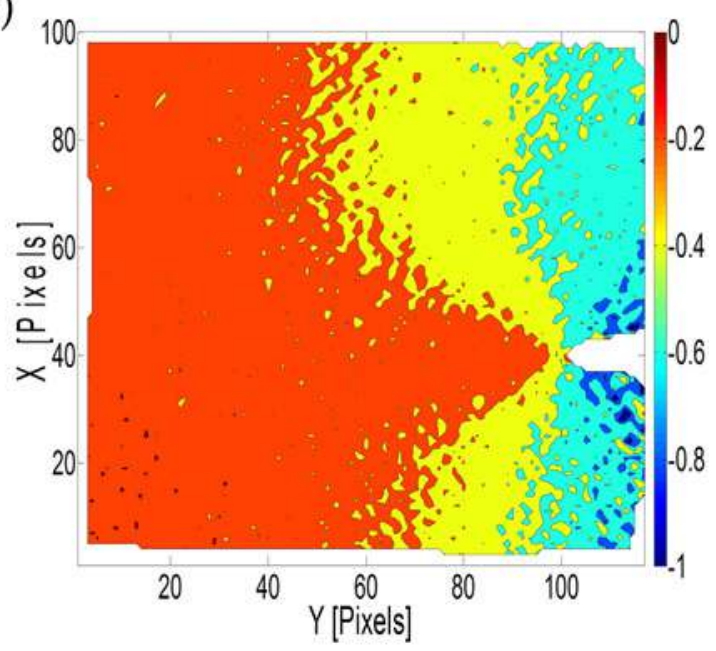

Fig. 4. Heterogeneity of stretch states at the end of the loading phase. a) Distribution in the $\left(I_{1}, I_{2}\right)$ plane. b) Distribution over the ROI. Colors range from red for UT to green for PS. c) Map of biaxiality ratios $b$. (For interpretation of the references to color in this figure legend, the reader is referred to the web version of this article.)

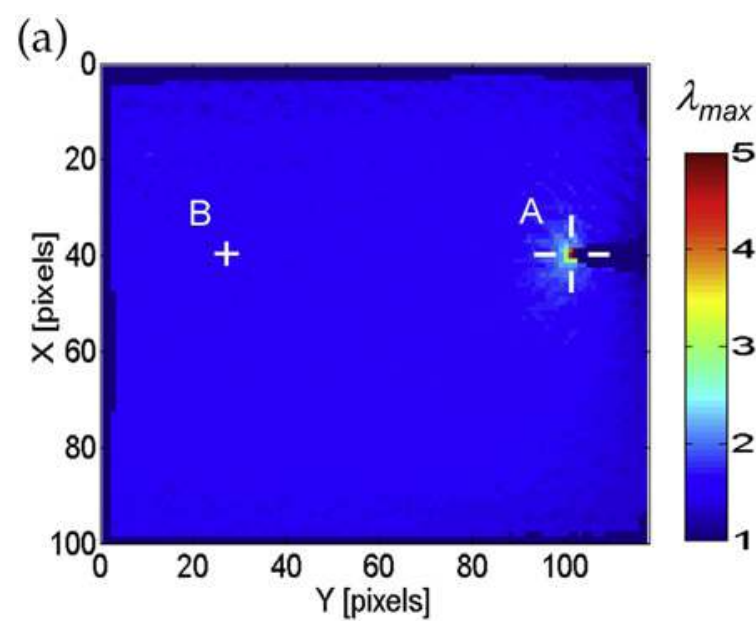

(b)

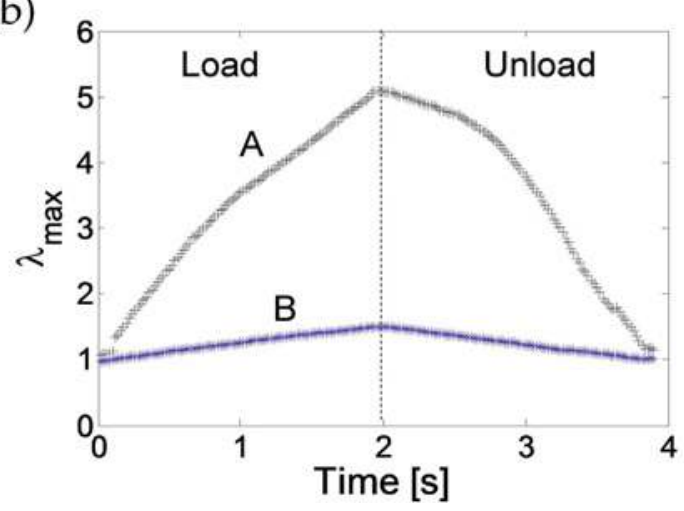

Fig. 5. Maximum stretch ratio $\lambda_{\max }$ a) Map over the ROI at the end of the loading phase. b) Variation at points $A$ and $B$.

zone of the highest values corresponds to the vicinity of the crack tip. The fact that the maximum value (equal to $8^{\circ} \mathrm{C} / \mathrm{s}$ ) is not exactly located at the crack tip but slightly shifted upwards is not interpretable in the present case, due to the fact that the spatial resolution is greater than the distance between the point at which the heat source is maximum and the crack tip. It can be noted that the size and the shape of high heat source zone around the crack tip are very close to those observed in terms of the crystallized zone in Ref. [3] (size) and Ref. [5] (size and shape).

Fig. 7-b gives the variation in the maximum value of the heat source near the crack tip and the corresponding maximum stretch ratio. The following comments can be made from this figure:

- during the loading phase, the heat source is positive and increases with the stretch ratio. The stronger increase which is observed from $t=1 \mathrm{~s}$ is mainly explained by the crystallization process. It should be noted that mechanical dissipation due to viscosity and through material softening also contributes to heat production. For instance, for the highest stretch ratios in SBR50, the sudden increase in the heat produced during loading is due to stress softening;

- during the unloading phase, the heat source remains positive for about $1 \mathrm{~s}$, then remains nearly equal to zero until the end. The entropic coupling heat source is expected to be negative since the strain rate is negative. Two reasons can be proposed to 
(a)

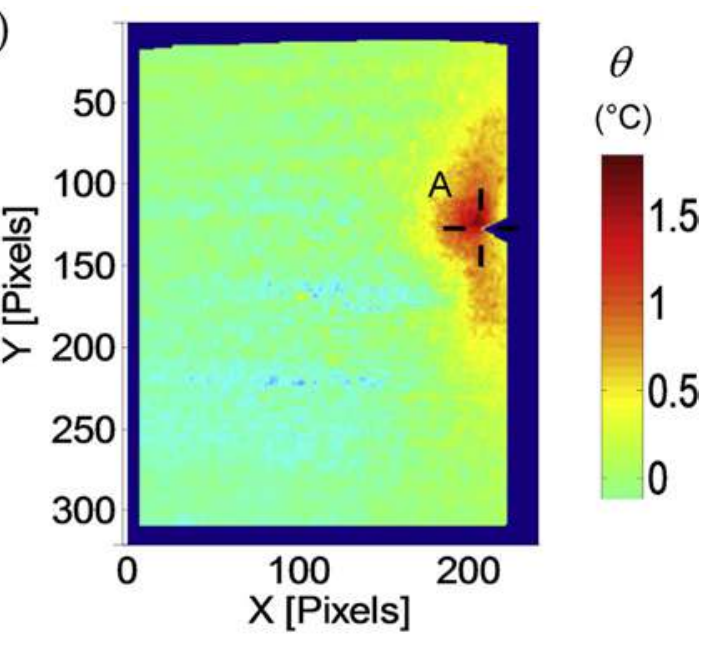

(b)

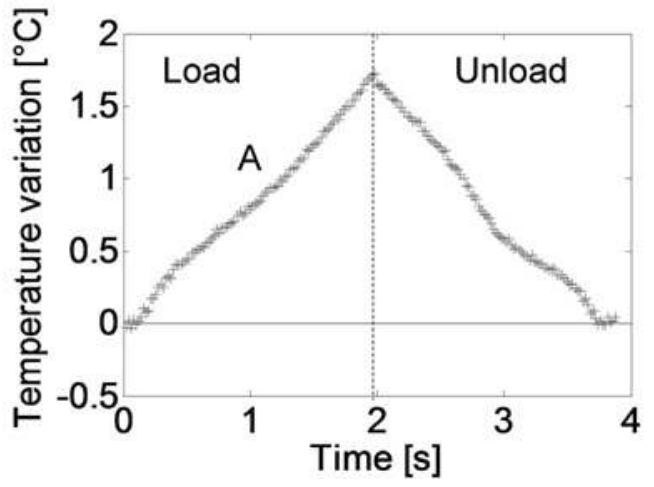

Fig. 6. Temperature variation $\theta$. a) Map over the ROI at the end of the loading phase. b) Variation at point $\mathrm{A}$.

explain the fact that the total heat source is positive. There is a significant production of mechanical dissipation due to viscosity (at least the same order of magnitude as the heat absorbed due to entropic coupling) and there is an additional crystallization. Indeed, the low value of the curve slope in the variation of $\lambda_{\max }$ may suggest that crystallization continues and produces heat [15]. Whatever the reason, there is the production of a positive heat source whose amplitude is higher than or equal to the entropic coupling. This result is important because it shows that the heat produced at a crack tip during a cycle is globally positive. This phenomenon may participate in the self-heating of cracked rubbers.

A similar analysis was recently performed for SBR50. The comparison with NR50 is presented in the next section.

\subsection{Comparison between NR50 and SBR50}

Fig. 8 shows the difference in the temperature variation maps between NR50 and SBR50 specimens at the end of the loading phase. The maximum value of $\theta$ is lower in the NR50 specimen than in the SBR50 specimen: $1.8{ }^{\circ} \mathrm{C}$ compared to $3.5^{\circ} \mathrm{C}$. This result was not really intuitive because the NR50 specimen exhibits a crystallization phenomenon, which produces additional heat. In fact, it can be explained by comparing the maximum stretch ratios $\lambda_{\max }$ at the crack tip.

Fig. 9-a shows the variation in $\lambda_{\max }$ at the crack tip for the two specimens. It can been seen that $\lambda_{\max }$ is lower in the NR50 specimen than in the SBR50 specimen: 5.1 compared to 9.5 at the end of (a)
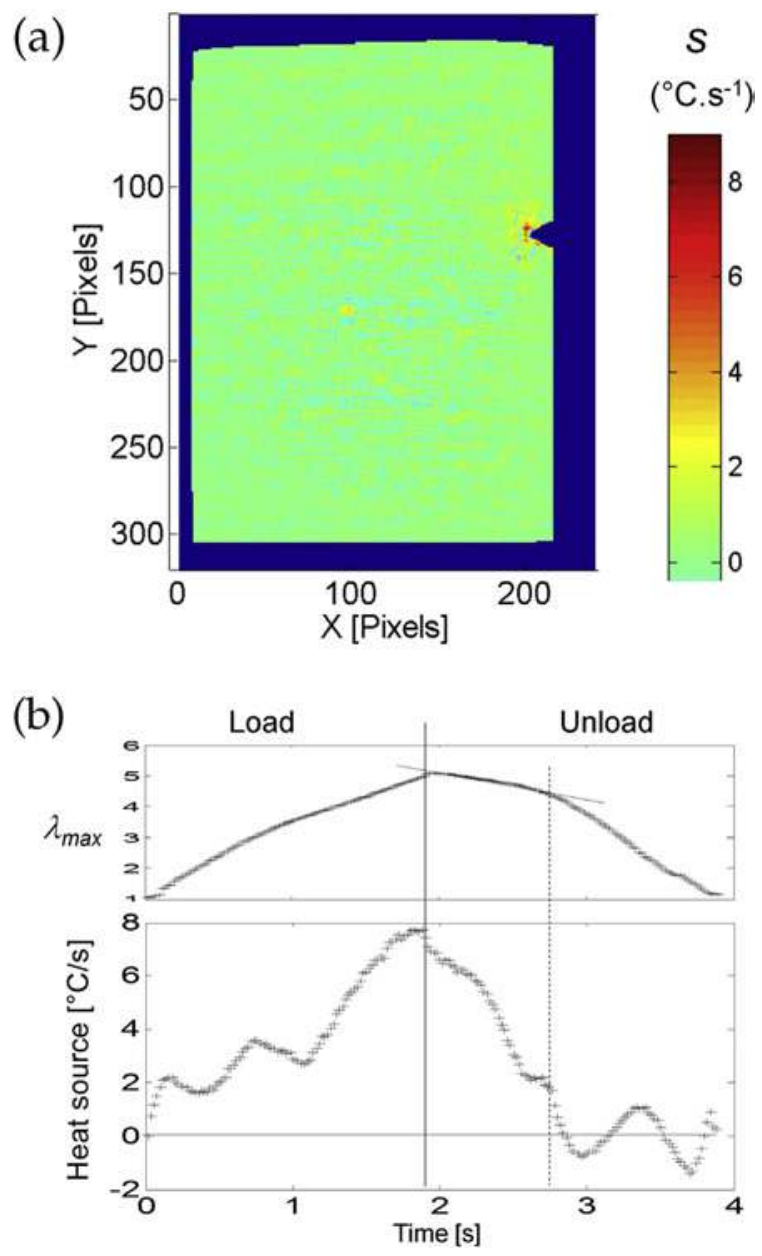

Fig. 7. Heat source $S$. a) Map over the ROI at the end of the loading phase. b) Variation in the maximum value of $S$ and the maximum stretch ratio (near the crack tip).

the loading phase. This can be explained by crystallization in the NR50 specimen, which increases the stiffness in the crack tip zone. Thus, in terms of heat production, crystallization acts in two opposite ways in the crack tip zone. On the one hand, crystallization locally produces additional heat. On the other hand, the increase in stiffness resulting from crystallization reduces the local level of stretching, thus reducing the quantity of heat produced by the material.

Some additional comments can be made about the comparison between the two types of specimen:

- Fig. 8 shows that the 'hot' zone is larger in the NR50 specimen than in the SBR50 specimen. This can be explained by the fact that crystallization occurs in a large zone around the crack tip in NR50 [3];

- Fig. 9-b shows that the heat source level at the crack tip is lower in the NR50 specimen than in the SBR50 specimen. For both specimens, the heat sources remain positive and high at the beginning of unloading. It can be noted that in the NR50 specimen, this phenomenon lasts longer. This can be explained by the continuation of the crystallization process;

- even though the maximum stretch ratios are different in NR50 and SBR50, the heat sources at the crack tip remain positive at the beginning of unloading. 


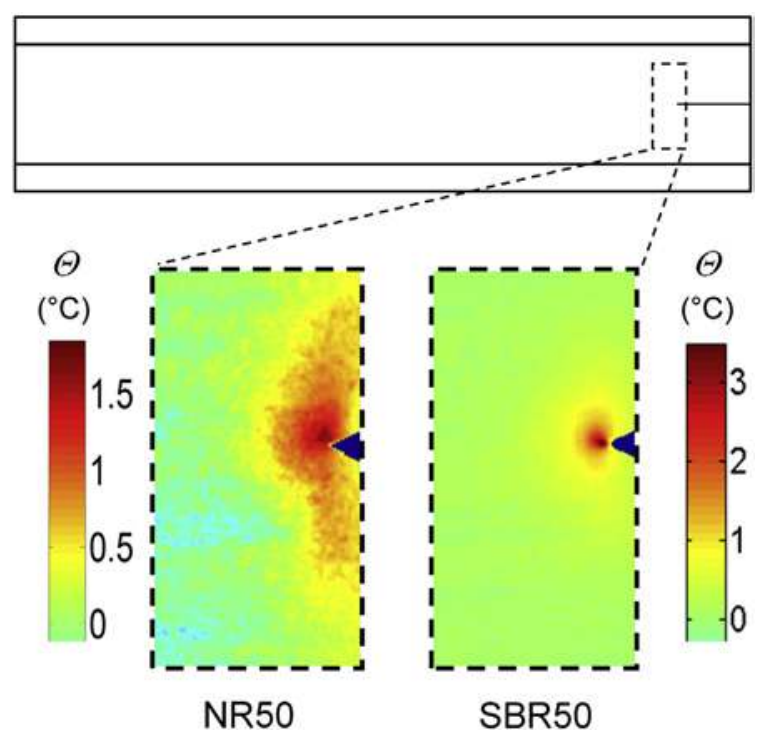

Fig. 8. Comparison between NR50 and SBR50 specimens: map of temperature variation near the crack tip at the end of the loading phase.

\section{Conclusion}

Infrared thermography and digital image correlation have been used simultaneously to analyze the thermomechanical behavior at the crack tip of a filled crystallizable rubber (NR50) and a filled noncrystallizable rubber (SBR50). Both specimens were subjected to the same load-unload mechanical cycle. Several results were obtained:
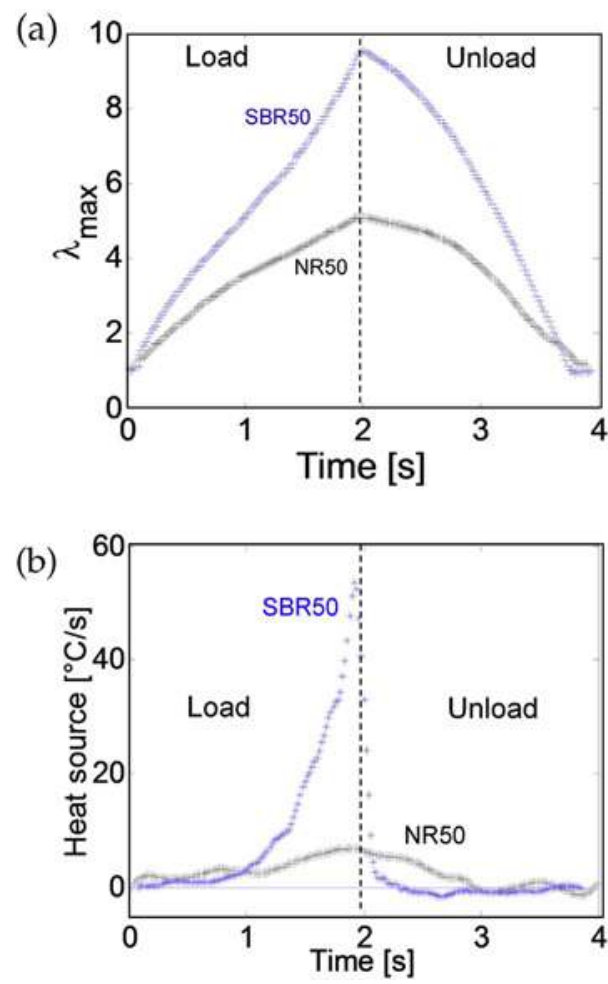

Fig. 9. Comparison between NR50 and SBR50 specimens: a) variation in the maximum stretch ratio and $b$ ) in the heat source near the crack tip.
- the calorimetric results showed that the heat produced in the crack tip zone is lower for the NR50 specimen than for SBR50. The 'hot' zone is larger in NR50, due to SIC.

- even though SIC produces additional heat, crystallites act as fillers and increase stiffness in the crack tip zone, which leads to a decrease in the stretch level in this zone. In other words, crystallization acts in two opposite ways in terms of heat production. This is an important consideration for the study of the self-heating of cracked rubbers subjected to fatigue loadings;

- it was observed that, similarly to non-crystallizable SBR50, the heat sources in the crack tip zone in NR50 remain positive during unloading. This was not observed for homogeneous tests (see Refs. $[13,16]$ ). This phenomenon is due to the production of mechanical dissipation (and to a continuation of crystallization at the beginning of unloading in the case of NR50) that is of the same order of magnitude as the heat source absorbed due to entropic coupling.

This study is the first one charactezing experimentally the heat source fields at the crack tip of elastomer rubbers. Further study is currently being carried out to link the location of the maximum heat source with the crack propagation path.

\section{Acknowledgments}

The authors thank the "Manufacture Française des pneumatiques Michelin" for supporting this study. We also thank J. Caillard for the fruitful discussions.

\section{References}

[1] Rivlin RS, Thomas AG. Rupture of rubber. I. Characteristic energy for tearing. J Polym Sci 1953;10:291-318.

[2] Andrews EH. A generalized theory of fracture mechanics. J Mater Sci 1974;9: 887-94.

[3] Trabelsi S, Albouy PA, Rault J. Stress-induced crystallization around a crack tip in natural rubber. Macromolecules 2002;35:10054-61.

[4] Bruening K, Heinrich G, Schneider K. Short-time behavior of strain-induced crystallization in natural rubber. In: GilNegrete N, Alonso A, editors. Constitutive models for rubber VIII; 2013. p. 457-60.

[5] Rublon P, Huneau B, Saintier N, Beurrot S, Leygue A, Verron E, et al. In situ synchrotron wide-angle X-ray diffraction investigation of fatigue cracks in natural rubber. J Synchrotron Radiat 2013;20:105-9.

[6] Bruening K, Schneider K, Roth SV, Heinrich G. Strain-induced crystallization around a crack tip in natural rubber under dynamic load. Polymer 2013;54: 6200-5.

[7] Le Cam JB, Huneau B, Verron E, Gornet L. Mechanism of fatigue crack growth in carbon black filled natural rubber. Macromolecules 2004:37:5011-7.

[8] Le Cam JB, Huneau B, Verron E. Description of fatigue damage in carbon black filled natural rubber. Fatigue Fract Eng Mater Struct 2008;31:1031-8.

[9] Zhang H, Scholz AK, Merckel Y, Brieu M, Berghezan D, Kramer EJ, et al. Strain induced nanocavitation and crystallization in natural rubber probed by rea time small and wide angle X-ray scattering. J Polym Sci Part B: Polym Phys 2013;51:1125-38.

[10] Le Cam JB, Toussaint E. The mechanism of fatigue crack growth in rubbers under severe loading: the effect of stress-induced crystallization. Macromolecules 2010;43:4708-14.

[11] Gabrielle B, Guy L, Albouy PA, Vanel L, Long DR, Sotta P. Effect of tear rotation on ultimate strength in reinforced natural rubber. Macromolecules 2011;44: 7006-15.

[12] Pottier T, Moutrille MP, Le Cam JB, Balandraud X, Grédiac M. Study on the use of motion compensation techniques to determine heat sources. Application to large deformations on cracked rubber specimens. Exp Mech 2009;49:561-74.

[13] Samaca Martinez JR, Le Cam JB, Balandraud X, Toussaint E, Caillard J. Filler effects on the thermomechanical response of stretched rubbers. Polym Test 2013;32:835-41.

[14] Samaca Martinez JR, Le Cam JB, Balandraud X, Toussaint E, Caillard J. Mechanisms of deformation in crystallizable natural rubber. Part 1: thermal characterization. Polymer 2013:54:2717-26.

[15] Samaca Martinez JR, Le Cam JB, Balandraud X, Toussaint E, Caillard J. Mechanisms of deformation in crystallizable natural rubber. Part 2: quantitative calorimetric analysis. Polymer 2013;54:2727-36.

[16] Samaca Martinez JR, Le Cam JB, Balandraud X, Toussaint E, Caillard J. New elements concerning the mullins effect: a thermomechanical analysis. Eur Polym J 2014;55:98-107. 
[17] Toussaint E, Balandraud X, Le Cam JB, Grédiac M. Combining displacement, strain, temperature and heat source fields to investigate the thermomechanical response of an elastomeric specimen subjected to large deformations. Polym Test 2012;31:916-25.

[18] Samaca Martinez JR, Toussaint E, Balandraud X, Le Cam JB, Caillard J. Heat and strain measurements at the crack tip of filled rubber under cyclic loadings using full-field techniques. Mech Mater 2014. http://dx.doi.org/10.1016/ j.mechmat.2014.09.011.

[19] Keating TJ, Wolf PR, Scarpace FL. Improved method of digital image correlation. Photogramm Eng Remote Sens 1975;41:993-1002.

[20] Sutton MA, Wolters WJ, Peters WH, Ranson WF, McNeill SR. Determination of displacements using an improved digital correlation method. Image Vis Comput 1983;1:133-9.
[21] Pan B, Qian KM, Xie HM, Asundi A. Two-dimensional digital image correlation for in-plane displacement and strain measurement: a review. Meas Sci Technol 2009;20:062001.

[22] Vacher P, Dumoulin S, Morestin F, Mguil-Touchal S. Bidimensional strain measurement using digital images. Proc Inst Mech Eng Part C 1999;213:811-7.

[23] Harris JG, Chiang YM. Nonuniformity correction of infrared image sequences using the constant-statistics constraint. IEEE Trans Image Process 1999;8:1148-61.

[24] Torres SN, Hayat MM. Kalman filtering for adaptive nonuniformity correction in infrared focal-plane arrays. J Opt Soc Am A 2003;20:470-80.

[25] Chrysochoos A, Huon V, Jourdan F, Muracciole JM, Peyroux R, Wattrisse B. Use of full-field digital image correlation and infrared thermography measurements for the thermomechanical analysis of material behaviour. Strain 2010;46:117-30. 